\title{
Terapia Cognitiva Comportamental para Insônia: Revisão Sistemática
}

\author{
Cognitive Behavioral Therapy for insomnia: systematic \\ review
}

\author{
Camila De Masi Teixeiraㄹ ${ }^{1}$ Lázaro Juliano Teixeira², Lucila Bizari \\ Fernandes do Prado ${ }^{3}$, Gilmar Fernandes do Prado ${ }^{3}$, Luciane \\ Bizari Coin de Carvalho ${ }^{4}$
}

\begin{abstract}
1.Psicóloga, Mestre, Departamento de Medicina, Unifesp, São Paulo-SP, Brasil.
2.Fisioterapeuta, Mestre, Departamento de Medicina, Unifesp, São Paulo-SP, Brasil.

3.Médico, Doutor, Departamento de Neurologia e Neurocirurgia, Unifesp, São Paulo-SP, Brasil.

4.Psicóloga, Doutora, Departamento de Neurologia e Neurocirurgia, Unifesp, São Paulo-SP, Brasil.
\end{abstract}

\section{Resumo}

Introdução. A Terapia Cognitivo-Comportamental (TCC) tem sido amplamente utilizada na prática clínica, e avaliada em ensaios clínicos e revisões sistemáticas, embora não existam protocolos bem definidos sobre o emprego das técnicas e sobre os desfechos que devem ser mensurados. Objetivo. o objetivo desta revisão sistemática foi avaliar a aplicação da TCC para adultos insones, comparada a quatro grupos de controle (lista de espera, sem tratamento, intervenção farmacológica e outras intervenções não farmacológicas), realizada em intervalo de quatro a oito semanas, utilizando as técnicas de Controle de Estímulos, Restrição de Sono, Reestruturação Cognitiva e Técnicas de Relaxamento. Método. As estratégias de busca foram submetidas às bases de dados MEDLINE (1966 a agosto de 2016), EMBASE (1980 a agosto de 2016), LILACS (1982 a agosto de 2016), Cochrane Central Register of Controlled Trials (agosto de 2016), PsycINFO (agosto de 2016) e WHO (agosto de 2016). Não houve restrição sobre a maneira de aplicação da TCC, e os resultados foram avaliados separadamente, quando necessário. Resultados. seis ensaios clínicos randomizados foram incluídos. A TCC apresentou melhora na qualidade de vida e na performance diurna, diminuiu os sintomas subjetivos da insônia e aumentou a eficiência do sono. Conclusão. Embora os resultados demonstrem efetividade da TCC, investigações posteriores são necessárias. Muitos resultados foram avaliados a partir da análise de um único estudo e a qualidade da evidência foi considerada como muito baixa.

Unitermos. Terapia Comportamental Cognitiva; Sono; Distúrbios do Início e da Manutenção do Sono; Revisão Sistemática.

\begin{abstract}
Introduction. Cognitive Behavioral Therapy (CBT) for insomnia has been used in clinical practice and evaluated in clinical trials and systematic reviews, although there are no set protocols for how the technique should be applied and results measured. Objective. The aim of this systematic review was to evaluate the application of CBT, incorporating the techniques of stimulus control, sleep restriction, cognitive restructuring, and relaxation techniques, when performed between 4 and 8 weeks for insomnia in adults compared to four different control groups: waitlist, no treatment, pharmacological intervention, and other nonpharmacological interventions. Method. The search strategies were found in MEDLINE (1966 to August 2016), EMBASE (1980 to August 2016), LILACS (1982 to August 2016), Cochrane Central Register of Controlled trials (August 2016), PsycINFO (data August 2016), and WHO (August 2016). There were no restrictions as to the manner of application of CBT, and the results were evaluated separately. Results. Six randomized controlled trials were included. CBT improved quality of life and daytime performance, reduced subjective symptoms of insomnia, and increased sleep efficiency. Conclusions. Although the results demonstrate effectiveness for CBT, further investigations are necessary, because most results were evaluated in terms of only one publication and the quality of evidence was considered very low.
\end{abstract}

Keywords. Cognitive Behavioral Therapy, Sleep, Insomnia, Psychological Treatment, Systematic Review. 
Trabalho realizado na Escola Paulista de Medicina (EPM) da Universidade Federal de São Paulo (Unifesp), São Paulo-SP, Brasil.

\section{INTRODUÇÃO}

A insônia é o distúrbio do sono mais comum e a queixa mais comum relacionada ao sono. Ela é definida como a percepção de má qualidade do sono ou sono não reparador, dificuldades para o início do sono, duração ou de consolidação, resultando em uma deficiência no funcionamento diurno do indivíduo ${ }^{1}$.

Entre os adultos, a insônia inclui a dificuldade para iniciar ou manter o sono, geralmente acompanhada de preocupações com longos períodos de vigília noturna ou quantidades insuficientes de sono noturno. Sintomas diurnos podem incluir fadiga, rebaixamento do humor ou aumento da irritabilidade, redução da motivação, energia ou iniciativa, sonolência, prejuízo do funcionamento social ou ocupacional e redução da qualidade de vida. Dores de cabeça, tensão muscular e dores também podem estar associadas à insônia. Em casos graves, a insônia pode aumentar o risco de acidentes de trabalho e de trânsito. A gravidade desse problema sugere que a diminuição do tempo de sono e a utilização de comprimidos para dormir estejam associados ao aumento da mortalidade.

De acordo com a Classificação Internacional de Distúrbios do Sono (ICSD), as causas mais comuns de 
insônia incluem doenças psiquiátricas ( $40 \%$ a $45 \%$ dos casos), distúrbios de sono relacionados ao ciclo circadiano (10\% a $15 \%$ dos casos) e distúrbio primário de sono (10 a $31 \%)^{1,2}$.

A prevalência média da insônia para a população geral é em torno de $35 \%$, sendo $10 \%$ a $15 \%$ dos casos classificados como moderado a severo. A prevalência da insônia é mais predominante em mulheres, e aumenta com a idade ${ }^{3}$.

Os indivíduos acometidos muitas vezes desenvolvem comportamentos que agravam e aumentam o problema, como passar muito tempo na cama, pensar em preocupações na hora de adormecer e manter uma rotina inadequada de horas de sono ${ }^{4}$.

Embora a terapia farmacológica seja comumente usada para o tratamento da insônia, seus efeitos aos pacientes tendem a diminuir ao longo do tempo ${ }^{5}$. A Terapia Cognitiva Comportamental (TCC) tem sido também amplamente utilizada para o tratamento da insônia, e há algumas evidências de que ela é eficaz em melhorar o sono e a qualidade de vida 6 .

A TCC é definida como uma forma de psicoterapia baseada na interpretação de situações (estrutura cognitiva de experiências) que determinam como um indivíduo se sente e se comporta. Em pacientes com insônia, a TCC normalmente envolve vários componentes, incluindo biofeedback, intenção paradoxal, instruções de controle de 
estímulo, restrição do sono, estratégias de relaxamento, reestruturação cognitiva e higiene do sono ${ }^{4,7-12}$.

Embora essas sejam as estratégias recomendadas pelos guidelines ${ }^{13}$, não existe um protocolo sobre quais técnicas devem ser aplicadas em conjunto. Nos últimos anos, estudos randomizados e revisões sistemáticas têm caracterizado as tentativas de demonstrar a evidência quanto ao número de sessões a serem realizadas e se a TCC deve ser administrada individualmente ou em grupos ${ }^{14}$, ${ }^{15}$, pessoalmente, por autoaplicação ${ }^{16}$ ou internet ${ }^{17}$.

Devido à grande variedade de técnicas de TCC e a limitações associadas a outras modalidades terapêuticas existentes, há a necessidade de uma revisão sistemática de ensaios clínicos randomizados centrada na TCC aplicada à insônia.

Nesse contexto, a presente revisão procura examinar a eficiência e segurança da TCC para insônia em adultos (acima de 18 anos), em comparação com quatro grupos controle (lista de espera, pacientes sem tratamento, intervenções farmacológicas e outras intervenções não farmacológicas).

$\mathrm{Na}$ hipótese de uma análise mais completa das intervenções em TCC para insônia, foram incluídos os estudos que realizaram atendimentos no intervalo de 4 a 8 semanas e que consideraram dentre as técnicas as orientações de controle de estímulos, restrição de sono, técnicas de relaxamento e reestruturação cognitiva. 
Não houve restrição sobre a maneira como a TCC foi aplicada nos grupos, pois os resultados foram analisados separadamente.

\section{MÉTODO}

O protocolo desta revisão foi aprovado pelo Comitê de Ética em Pesquisa da Universidade Federal de São Paulo, Brasil, sob o número de parecer 816.776.

\section{Pesquisa bibliográfica}

Foram pesquisadas as seguintes bases de dados: MEDLINE (1966 a agosto de 2016), EMBASE (1980 a agosto de 2016), LILACS (1982 a agosto de 2016), Cochrane Central Register of Controlled Trials (agosto de 2016), PsycINFO (agosto de 2016), e WHO (agosto de 2016). Uma detalhada estratégia de busca foi desenvolvida para cada uma das bases de dados, considerando-se as diferenças de vocabulário e sintáticas. As buscas foram realizadas de maneira abrangente, de modo a reunir o maior número possível de estudos.

Todos os artigos potencialmente relevantes foram avaliados por meio de um formulário previamente preparado, baseado nos critérios de inclusão. Dois pesquisadores avaliaram a relevância de cada artigo de forma independente e em dupla. Discordâncias em relação à seleção dos estudos foram resolvidas por um terceiro pesquisador. A extração dos dados foi realizada pelos 
mesmos pesquisadores que avaliaram a possibilidade de inclusão dos artigos, e então foi realizada a análise dos dados.

Os dados foram obtidos por meio de fichas de extração que incluíam informações relativas a características dos participantes (país de origem, tamanho da amostra, idade, sexo, critérios de diagnóstico, história, local, participantes após randomização, e proporção de perdas de seguimento), detalhes de intervenção, intervenção controle (lista de espera, sem tratamento, intervenções farmacológicas ou outras intervenções não farmacológicas), e as medidas dos desfechos primários e secundários de interesse e seus valores numéricos.

\section{Tipos de Estudo}

Todos os ensaios clínicos randomizados e quasirandomizados foram incluídos. Ensaios clínicos quasirandomizados foram definidos como aqueles que utilizaram alocação inadequada, como data de nascimento, dia da semana ou mês do ano, número do prontuário ou aqueles que simplesmente alocaram os participantes ${ }^{18}$.

Ensaios clínicos que realizaram as intervenções em menos de quatro semanas foram excluídos.

\section{Participantes}

Participantes com idade acima de 18 anos, diagnosticados com insônia crônica pelos critérios definidos pelos autores dos estudos primários (clínicos e/ou 
polissonográfico). Na insônia, o monitoramento da polissonografia pode demonstrar os seguintes aspectos: redução da eficiência do sono, redução do estágio N3 e latência de sono aumentada. O diagnóstico clínico da insônia considerou os critérios diagnósticos padrão, como do Manual Diagnóstico e Estatístico dos Transtornos Mentais (DSM) ${ }^{19}$, Classificação Internacional dos Distúrbios de Sono (ICSD) ${ }^{1}$, e Classificação Internacional de Doenças $(C I D)^{20}$ ou simplesmente indivíduos com queixas e sinais clínicos de insônia.

Estudos com participantes que apresentaram comorbidades como distúrbios do sono relacionados ao movimento, distúrbios respiratórios e distúrbios neurológicos ou psiquiátricos foram excluídos.

Todos os cenários, vinculados a ambientes de ensino e pesquisa, foram considerados.

\section{Tipo de Intervenção}

\section{Grupo Intervenção}

Foram incluídos artigos que avaliaram a TCC aplicada ao tratamento da insônia. Não houve restrição quanto ao modo específico de aplicação da TCC nos estudos quanto ao método (presencialmente, via internet ou material impresso, individualmente ou em grupos). A intervenção precisou ser realizada no intervalo de quatro a oito semanas, ter incluído no protocolo as técnicas que seguem:

Instruções de Controle de Estímulos: são baseadas nos princípios de condicionamento clássico, em que a cama 
deve ser utilizada apenas para o sono. O objetivo desta técnica é re-associar o estímulo do quarto ao início rápido do sono. Determinando um horário fixo para o despertar, a estratégia estabiliza o ciclo sono/vigília. As instruções incluem: 1) Ir para a cama apenas quando sentir sono; 2) Uso da cama apenas para o sono e atividade sexual (única exceção); 3) Deixar a cama, sair do quarto e iniciar atividade monótona e que induza o relaxamento se não adormecer após 15 minutos da primeira instrução. Retornar para a cama apenas quando se sentir sonolento novamente, e repetir o procedimento o quanto for necessário; 4) Evitar ler, assistir televisão, trabalhar ou comer na cama; 5) Acordar e levantar-se diariamente no mesmo horário, inclusive aos finais de semana; 6) Evitar cochilos diurnos $10,21,22$.

Restrição de sono: visa consolidar o sono, reduzindo a quantidade de tempo que as pessoas passam na cama, aumentando a eficiência do sono. A primeira etapa envolve a restrição do tempo permitido na cama para igualar a quantidade média de tempo que a pessoa gasta realmente dormindo. No final de cada semana, o índice de eficiência do sono (ES) é calculado (tempo gasto no sono / tempo passado na cama $\times 100)$. Se a ES for superior a $85 \%$, um adicional de 15 a 20 minutos de tempo na cama é adicionado à noite. Se a ES for inferior a $85 \%$, o tempo na cama é ainda mais restringido por 15 a 20 minutos. Reduzir o tempo na cama para menos de 5,5 horas não é 
geralmente recomendado. A restrição do sono requer tremenda disciplina ${ }^{23,24}$.

Métodos de Relaxamento: os mais estudados e utilizados no tratamento de insônia são: relaxamento muscular progressivo, biofeedback, imaginação guiada e respiração diafragmática (abdominal). O relaxamento muscular progressivo é realizado tensionando e relaxando uma série de músculos, progressivamente. Biofeedback pode ajudar no relaxamento, fornecendo pistas visuais ou auditivas para informar a pessoa se $O$ alvo correlato fisiológico de relaxamento foi atingido. A técnica de imaginação guiada ajuda a pessoa a imaginar vividamente uma imagem neutra ou agradável. A formação dessas imagens pode induzir diretamente a um estado de relaxamento ou indiretamente desviar a atenção de um estímulo desencadeador de ansiedade. A respiração diafragmática (abdominal) facilita a desaceleração da respiração, levando a um estado de relaxamento. É fácil de aprender e pode induzir o relaxamento e o sono de forma relativamente rápida. O relaxamento deve ser introduzido e praticado com o objetivo de reduzir os níveis de excitação, e não como um meio para induzir o sono. Métodos de relaxamento não requerem ajudas externas, tais como fitas de áudio ou aparelhos de som ${ }^{2} 25$.

Reestruturação Cognitiva: é usada para identificar os pensamentos disfuncionais e atitudes que as pessoas podem ter sobre o sono e substituí-los por pensamentos e comportamentos mais adaptativos. A reestruturação 
cognitiva em longo prazo pode ser usada para superar todas as preocupações a respeito do sono e eliminar ansiedades associadas ao sono ruim ou à incapacidade de adormecer $2,4,26$.

Intervenções de biofeedback e intenção paradoxal não foram consideradas porque são menos utilizadas nos protocolos. A técnica de higiene do sono, embora não seja considerada parte da TCC nas diretrizes do tratamento padrão da insônia propostas pelos guidelines, foi utilizada em quase todos os grupos e relatada em quase todos os artigos.

\section{Grupo Controle}

O grupo controle considerado foi Lista de Espera ou Tratamento Usual (LE), Sem Tratamento (ST), Intervenções Farmacológicas (IF) - como benzodiazepínicos, agentes dopaminérgicos, antidepressivos, analgésicos e outros - e Outras Intervenções Não Farmacológicas (OINF) (ioga, meditação e outros). Embora os grupos LE e ST tenham características semelhantes, os pacientes de LE podem manter contato com os pesquisadores, o que facilita 0 controle das variáveis, tais como diários de sono e / ou outras avaliações clínicas enquanto aguardam tratamento. Os doentes atribuídos a grupos ST podem ter sido submetidos a qualquer outro tratamento sem que os experimentadores estivessem cientes. Portanto, LE e ST foram analisados separadamente, conforme o protocolo. 


\section{Caracterização dos Riscos de Viés nos Artigos Incluídos}

Dois autores avaliaram a qualidade dos artigos de forma independente. Discordâncias foram resolvidas por discussão. O Método foi baseado no Manual Cochrane de Revisões Sistemáticas de Intervenções ${ }^{18}$. Os critérios considerados foram: geração de sequência de randomização adequada; ocultação da alocação; mascaramento dos participantes e pesquisadores; cegamento de avaliação de resultados; dados de resultados incompletos; relatório seletivo de resultados e outros vieses. Cada critério foi avaliado como "baixo risco", "alto risco", ou "risco incerto". O "risco incerto" foi indicado quando não havia informação suficiente para permitir um julgamento claro.

Usamos a classificação GRADE para avaliar a qualidade da evidência por meio dos resultados. Os estudos primários incluídos foram classificados no mais alto nível, e foram rebaixados devido às seguintes limitações do estudo (risco de viés): limitações de concepção, de inconsistência (grande heterogeneidade), indiretividade de provas (comparações ou desfechos avaliados sem que o estudo tenha sido proposto a eles), imprecisão (estudos realizados com poucos participantes ou efeito acima de 0,5 ) e viés de publicação (poucos estudos para análise da evidência).

Essa classificação especifica quatro graus de qualidade da evidência: 1) Alta qualidade: Futuras pesquisas não são necessárias para aumentar a confiança no efeito estimado. 
2) Qualidade moderada: Pesquisas são recomendadas para aumentar a confiança no efeito, e podem mudar as estimativas. 3) Baixa qualidade: alta recomendação para novas pesquisas, que devem alterar estimativas. 4) Qualidade muito baixa: muita incerteza sobre as estimativas. Novas pesquisas são necessárias.

\section{Desfechos e medidas}

Melhora na qualidade do sono, redução das queixas e sintomas subjetivos (auto-relato de satisfação do sono), e performance diurna foram considerados como desfechos primários e analisados por diários de sono ou escalas.

Como desfechos secundários foram considerados latência do início do sono (medido como o tempo necessário para adormecer), eficiência do sono (medida como a proporção de tempo dormindo / tempo na cama), vigília após o início do sono (medido em unidades de tempo), redução de despertares, tempo total de sono (medida em unidades de tempo), redução da frequência do consumo de drogas hipnóticas e redução de efeitos adversos relacionados ao uso de fármacos. Todos os resultados foram analisados como foram apresentados, por diário do sono ou polissonografia.

O nível de ansiedade foi medido por meio do Inventário Beck de Ansiedade $(B A I)^{27}$ e o nível de depressão foi medido pelo Inventário Beck de Depressão $(B D I)^{28}$. A qualidade de vida foi mensurada através do SF$36^{29}$. 


\section{Análise Estatística}

Nesta revisão sistemática, o método usado para avaliar o conjunto de resultados de dois ou mais estudos foi a meta-análise. A heterogeneidade foi classificada conforme os valores dos testes $\mathrm{I}^{2}$ e Qui-quadrado, e foi considerado valor de significância para $P<0,05$. Quando foi verificada heterogeneidade significativa, tentou-se explicar as diferenças em função das características clínicas dos estudos incluídos. A heterogeneidade estatística foi avaliada assumindo grande heterogeneidade quando $\mathrm{I}^{2}>50 \%$.

\section{RESULTADOS}

A pesquisa nas bases de dados identificou um total de 2.768 artigos potencialmente relevantes, e 34 artigos foram identificados por meio de uma busca manual.

As revisões sistemáticas (53 registros) foram excluídas. Após examinar títulos e resumos e após a remoção de duplicatas (631 registros) foram identificados 184 artigos potencialmente relevantes. Desses, 173 foram excluídos, três estão aguardando dados (Arnedt 201330, Bjortvan 201131, Espie 200132) e dois estão à espera de tradução (Fornal-Pawlowska $2013^{33}$ e Ueda 2008 ${ }^{34}$ ). Seis artigos preencheram os critérios de inclusão para a análise principal dessa revisão e foram analisados (Figura 1). 


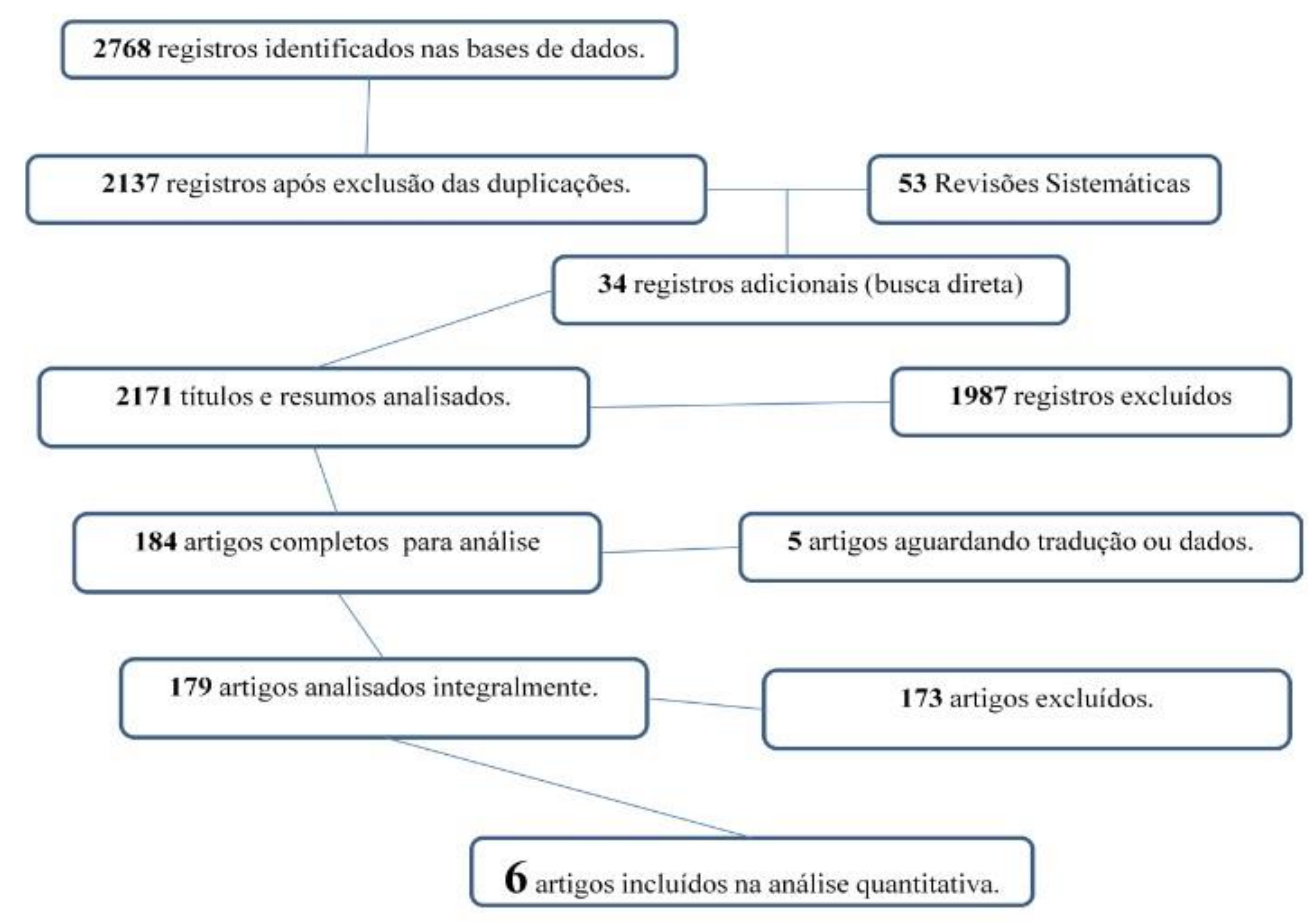

Figura 1. Diagrama de seleção dos artigos.

Todos os artigos incluídos validaram suas pesquisas junto às instituições responsáveis por avaliar os princípios éticos de pesquisa com seres humanos.

Os seis artigos incluídos nessa revisão foram: Espie 201235, Jacobs 200436, Omvik 200837, Strom 200426, Sivertsen $2006^{38}$ e Van Straten $2009^{39}$. As características dos artigos incluídos encontram-se na Tabela 1.

A comparação da TCC contra os quatro grupos controle estabelecidos no protocolo resultou nas seguintes análises: Foram identificados três artigos (Strom 200426, Van Straten 200939, e Espie 2012 ${ }^{35}$ ) comparando TCC $(n=235)$ com LE $(n=230)$, três artigos (Omvik 200836, Jacobs $2004^{37}$ e Sivertsen $\left.2006^{38}\right)$ comparando TCC $(n=56)$ com IF $(n=53)$ e um artigo (Espie $2012^{35}$ ) comparando TCC $(n=55)$ com OINF $(n=55)$ (construção de imagens 


\begin{tabular}{|c|c|c|c|c|c|c|c|}
\hline & $\mathbf{N}$ & $\begin{array}{c}\text { Pais/ } \\
\text { Universidade/ } \\
\text { Participantes }\end{array}$ & $\begin{array}{c}\text { Idade } \\
\text { (média em } \\
\text { anos) } / \% \\
\text { mulheres }\end{array}$ & $\begin{array}{c}\text { Critério } \\
\text { Diagnóstico }\end{array}$ & Design & Follow up & Dados acessados \\
\hline $\begin{array}{c}\text { Espie } \\
2012^{35}\end{array}$ & 164 & $\begin{array}{l}\text { Reino Unido } \\
\text { Participantes de } \\
\text { comunidade }\end{array}$ & $49 / 73,2 \%$ & DSM-V & $\begin{array}{l}\text { TCC }(\mathbf{n}=\mathbf{5 5}) \text { Educação sobre Sono, } \\
\text { Relaxamento, Controle de Estímulos, Restrição } \\
\text { de Sono, Técnicas Cognitivas (Reestruturação, } \\
\text { Paradoxal, Mindfulness, Imagens, Parada de } \\
\text { Pensamento). Seis sessões, minimo em seis } \\
\text { semanas. Ministrado por terapeuta. Sem contato } \\
\text { face a face. } \\
\text { Treino Imaginativo }(\mathbf{n}=\mathbf{5 5}) \text { Educação sobre } \\
\text { sono, Desenvolvimento de Hierarquia, } \\
\text { Treinamento Imaginário, } \\
\text { Rseudodessensibilização, Controle Respiratório. } \\
\text { Seis sessões, minimo de seis semanas. Ministrado } \\
\text { por terapeuta. Sem contato face a face. } \\
\text { Tratamento Usual (n = 54) Orientação de que o } \\
\text { tratamento eficaz seria oferecido ao final do } \\
\text { estudo. Diário de sono gravado. Sem contato face } \\
\text { a face. }\end{array}$ & 8 sepmanas & $\begin{array}{l}\text { ES / LS / DAIS / TTA } \\
\text { TTS } \\
\text { Impacto no funcionamento } \\
\text { diurno e na performance } \\
\text { (concentração, } \\
\text { produtividade, humor, } \\
\text { relacionamentos e } \\
\text { energia). Qualidade do } \\
\text { Sono. }\end{array}$ \\
\hline $\begin{array}{l}\text { Jacobs } \\
2004^{36}\end{array}$ & 63 & $\begin{array}{c}\text { EUA / } \\
\text { Voluntários - } \\
\text { Beth Israel } \\
\text { Deaceness } \\
\text { Medical Center } \\
\text { em Boston }\end{array}$ & $\begin{array}{l}47,05 / \\
69,8 \%\end{array}$ & $\begin{array}{c}\text { ICSD e DSM } \\
\text { IV }\end{array}$ & $\begin{array}{l}\text { TCC }(\mathbf{n}=15) \text { Intervenções cognitivas, } \\
\text { comportamentais (Controle de estimulos, } \\
\text { restrição de sono), relaxamento e educacionais } \\
\text { que influem sobre a latência do sono. } \\
\text { Farmacoterapia }(n=15) \text {. Zolpidem, } 30 \text { minutos } \\
\text { antes de deitar. } \\
\text { TCC + Farmacoterapia }(n=18) \text { Zolpidem + } \\
\text { TCC } \\
\text { Placebo }(n=15) \text { Cápsulas gelatinosas idênticas }\end{array}$ & $\begin{array}{l}1,3,6,12 \\
\text { meses }\end{array}$ & ES / LS / DAIS / TTS \\
\hline $\begin{array}{l}\text { Omvik } \\
2008^{37}\end{array}$ & 46 & $\begin{array}{l}\text { Noruega/ } \\
\text { Voluntários }\end{array}$ & $\begin{array}{r}60,9 / \\
47,8 \%\end{array}$ & $\begin{array}{l}\text { DSM IV e } \\
\text { PSG }\end{array}$ & $\begin{array}{l}\text { TCC }(\mathbf{n}=\mathbf{2 3}) \text { Controle de Estimulos, Restrição } \\
\text { de Sono, Terapia Cognitiva, Relaxamento } \\
\text { Progressivo. } \\
\text { Farmacoterapia }(\mathbf{n}=\mathbf{2 2}) \text { Zopiclone }(7,5 \mathrm{mg}), 30 \\
\text { minutos antes de deitar. } \\
\text { Placebo }(\mathbf{n}=\mathbf{1 2} \text { ) Cápsulas gelatinosas idênticas }\end{array}$ & 6 meses & $\begin{array}{l}\text { Escalas de Sintomas } \\
\text { Psicológicos } \\
\text { (Preocupação, Ansiedade } \\
\text { Depressão). } \\
\text { Alerta e } \\
\text { Vigilância } \\
\text { Qualidade de Vida }\end{array}$ \\
\hline $\begin{array}{l}\text { Sivertsen } \\
2006^{38}\end{array}$ & 46 & $\begin{array}{l}\text { Noruega/ } \\
\text { Ambulatório }\end{array}$ & $\begin{array}{r}60,8 \\
47,8 \%\end{array}$ & $\begin{array}{l}\text { Queixa } \\
\text { clinica de } \\
\text { insônia e } \\
\text { DSM TV }\end{array}$ & $\begin{array}{l}\text { TCC }(\mathbf{n}=18) \text { Controle de Estimulos, Restrição } \\
\text { de Sono, Terapia Cognitiva, Relaxamento } \\
\text { Progressivo. } \\
\text { Farmacoterapia }(\mathbf{n}=16) \text { Zopiclone }(7,5 \mathrm{mg}) \\
\text { Placebo }(\mathbf{n}=12) \text { Cápsulas gelatinosas idênticas }\end{array}$ & 6 meses & $\begin{array}{l}\text { TTS/TTA/ES } \\
\text { Sono de Ondas Lentas }\end{array}$ \\
\hline $\begin{array}{c}\text { Strom } \\
2004^{26}\end{array}$ & 109 & $\begin{array}{c}\text { Suécia / } \\
\text { Voluntários }\end{array}$ & $\begin{array}{l}44,1 / \\
65,1 \%\end{array}$ & $\begin{array}{l}\text { DSM IV e } \\
\text { ASDA }\end{array}$ & $\begin{array}{l}\text { TCC }(\mathbf{n}=\mathbf{5 4}) \text { Controle de Estimulos, Restrição } \\
\text { de Sono, Reestruturação Cognitiva, Informações } \\
\text { sobre Desmame de Medicação, Relaxamento. } \\
\text { Lista de Espera }(\mathbf{n}=\mathbf{5 5})\end{array}$ & 9 meses & $\begin{array}{c}\text { LS / DAIS / TTA / TTS / } \\
\text { TC / ES / Pensamentos e } \\
\text { Atitudes } \\
\text { (DBAS) } \\
\text { Quantidade de } \\
\text { despertares } \\
\text { Qualidade do } \\
\text { Sono } \\
\text { Despertar cedo } \\
\end{array}$ \\
\hline $\begin{array}{c}\text { Van } \\
\text { Straten } \\
2009^{39}\end{array}$ & 247 & $\begin{array}{l}\text { Paises baixos / } \\
\text { Voluntários }\end{array}$ & $52 / 65,9 \%$ & $\begin{array}{l}\text { Queixa } \\
\text { clinica de } \\
\text { insônia }\end{array}$ & $\begin{array}{l}\text { TCC }(\mathbf{n}=126) \text { Livro de TCC (Controle de } \\
\text { Estimulos, Restrição de sono, Terapia Cognitiva) } \\
+ \text { Áudio (Relaxamento Progressivo). } \\
\text { Lista de Espera }(\mathbf{n}=121)\end{array}$ & 1 & $\begin{array}{l}\text { TTS / LS / } \\
\text { QS Qualidade do } \\
\text { Sono Ansiedade e } \\
\text { Depressão } \\
\text { Pensamentos e Atitudes } \\
\text { (DBAS) } \\
\text { Qualidade de Vida }\end{array}$ \\
\hline
\end{tabular}

Tabela 1. Característica dos artigos incluídos. 
agradáveis como placebo). Nenhum artigo que comparasse TCC com o grupo ST foi encontrado.

Todos os artigos encontrados aplicaram as quatro técnicas previstas no protocolo, embora o procedimento da TCC tenha sido realizado de maneira diferente. Strom $2004^{26}$ e Espie $2012^{35}$ administraram a TCC via internet, e seus resultados, quando mensurados com as mesmas escalas para os mesmos desfechos, puderam ser agrupados.

Omvik 200837, Jacobs $2004^{36}$ e Sivertsen $2006^{38}$ administraram a TCC em sessões individuais e, novamente, seus resultados quando mensurados com as mesmas escalas para os mesmos desfechos puderam ser agrupados. Van Straten $2009^{39}$ foi o único que aplicou a TCC por meio de materiais bibliográficos impressos e em DVD para que os participantes os utilizassem em casa.

\section{Viés nos Artigos Incluídos}

Geração de sequência aleatória e Alocação (viés de seleção)

Dentre os artigos incluídos, Omvik $2008^{37}$ e Strom $2004^{26}$ não descreveram o modo de geração de sequência e randomização aleatória dos participantes. Nenhum estudo apresentou alto risco de viés em relação à alocação, mas Omvik $2008^{37}$ não descreveu esta informação, e o viés foi considerado como "incerto". Espie 2012 ${ }^{35}$, Jacobs $2004^{36}$, Sivertsen 2006 38 , Strom $2004^{26}$ e van Straten $2009^{39}$ descreveram o processo de alocação dos participantes, indicando um baixo risco de viés. 


\section{Cegamento (viés de desenho do estudo)}

Em van Straten $2009^{39}$, os participantes foram informados sobre o resultado da randomização antes de iniciar o tratamento; este foi considerado um possível risco de parcialidade, e considerado como incerto. Os outros cinco estudos incluídos foram classificados como baixo risco de viés devido à descrição explícita do cegamento dos participantes e pesquisadores.

Cegamento da avaliação dos resultados (viés de detecção)

Dois artigos não forneceram informações sobre 0 cegamento da avaliação de resultados, e assim foram classificados como de risco de viés "incerto" (Espie $2012^{35}$ e Jacobs $\left.2004^{36}\right)$.

Resultado com Dados Incompletos (viés de perda) e Relato Seletivo

Dentre os artigos incluídos, Strom $2004^{26}$ e Jacobs $2004^{36}$ apresentaram risco de viés de perda. Apesar de descreverem a quantidade e os motivos de participantes que evadiram os grupos em que estavam alocados durante os estudos, estes não foram contabilizados na análise final dos resultados. Strom $2004^{26}$ por ter perdido ao longo do estudo $28 \%$ dos participantes, teve um alto risco de viés considerado. 


\section{Desfechos e Qualidade das Evidências}

Vários instrumentos foram utilizados para mensurar os desfechos nas diferentes comparações, e os estudos foram agrupados, quando possível, considerando seus meios para medir os resultados. Os desfechos foram avaliados nesta revisão a partir de variáveis contínuas, pois foi o meio utilizado pelos artigos incluídos para expressão dos resultados. Todos os resultados avaliados nas três comparações estão expressos na Tabela 2.

A melhora na Qualidade do Sono foi avaliada com escalas analógicas de 1 a 5 (Strom 200426), 1 a 10 (Van Straten 200939), 1 a 100 (Espie 2012 ${ }^{35}$ ) e por meio do Sleep Condition Indicator (SCI) (Espie 2012 ${ }^{35}$ ). O estudo de Espie 201235, comparando TCC com LE demonstrou melhora na qualidade do sono no grupo de intervenção TCC, na escala de 1 a 100 (MD 12,30; IC 5,28 a 19,32; $p=0,0006$ ) e através do SCI (MD 2,52; IC 2,41 a 2,63; $p<0,00001)$. Resultados similares foram reportados na comparação da TCC vs. OINF (MD 1,82; IC 1,71 a 1,93; $p<0,00001)$. Apesar dos resultados favoráveis, a qualidade da evidência foi considerada muito baixa.

Performance diurna foi mensurada por meio do estado de alerta, tempo de reação e número de respostas corretas no teste Vigil 5.0 (Omvik 200837). A capacidade de se manter acordado, concentração, produtividade, humor, relacionamentos e energia foram avaliados em uma escala analógica de 0 a 4 (Espie 2012 ${ }^{35}$ ). Nos níveis de concentração, produtividade e capacidade de se manter 


\begin{tabular}{|c|c|c|c|c|c|c|}
\hline \multicolumn{7}{|c|}{ Comparação TCC x Lista de Espera } \\
\hline Desfecho ou subgrupo & Artigo & $\begin{array}{c}\text { Tipo de } \\
\text { aplicação da } \\
\text { TCC }\end{array}$ & Medida & $\mathbf{N}$ & $\begin{array}{l}\text { Efeito } \\
\text { estimado }\end{array}$ & $\begin{array}{l}\text { Desfecho } \\
\text { favorável } \\
\text { para: }\end{array}$ \\
\hline Melhor qualidade do sono & $\begin{array}{l}\text { Strom } \\
2004^{26}\end{array}$ & \multirow{3}{*}{ Internet } & Escala 1 a 5 & 81 & $\begin{array}{c}0.11 \\
{[-0,17 ; 0,39]}\end{array}$ & TCC \\
\hline Melhor qualidade do sono & $\begin{array}{l}\text { Espie } \\
2012^{35}\end{array}$ & & $\begin{array}{l}\text { Escala } \\
1 \text { a } 100\end{array}$ & 109 & $\begin{array}{c}12,3 \\
{[5,28 ; 19,32]}\end{array}$ & TCC \\
\hline Melhor qualidade do sono & $\begin{array}{l}\text { Espie } \\
2012^{35} \\
\end{array}$ & & $\mathrm{SCI}$ & 109 & $\begin{array}{c}2,52 \\
{[2,41 ; 2,63]}\end{array}$ & TCC \\
\hline Melhor qualidade do sono & $\begin{array}{l}\text { Van } \\
\text { Straten } \\
2009^{39}\end{array}$ & Autoaplicação & $\begin{array}{l}\text { Escala } \\
1 \text { a } 10\end{array}$ & 234 & $\begin{array}{c}0,00 \\
{[-0,27 ; 0,27]}\end{array}$ & $\begin{array}{c}\text { Sem } \\
\text { diferença }\end{array}$ \\
\hline $\begin{array}{l}\text { Redução dos sintomas } \\
\text { subjetivos da insônia }\end{array}$ & $\begin{array}{l}\text { Van } \\
\text { Straten } \\
2009^{39}\end{array}$ & Autoaplicação & SEF & 247 & $\begin{array}{c}2,30 \\
{[1,61 ; 2,99]}\end{array}$ & TCC \\
\hline $\begin{array}{l}\text { Redução dos sintomas } \\
\text { subjetivos da insônia }\end{array}$ & $\begin{array}{l}\text { Strom } \\
2004^{26}\end{array}$ & Internet & DBAS & 65 & $\begin{array}{c}-14,40 \\
{[-23,13 ;-5,7]}\end{array}$ & TCC \\
\hline $\begin{array}{l}\text { Redução dos sintomas } \\
\text { subjetivos da insônia }\end{array}$ & $\begin{array}{l}\text { Van } \\
\text { Straten } \\
2009^{39}\end{array}$ & Autoaplicação & DBAS & 247 & $\begin{array}{c}10,00 \\
{[6,65 ; 13,35]}\end{array}$ & Lista espera \\
\hline $\begin{array}{l}\text { Performance diurna } \\
\text { (concentração, } \\
\text { produtividade, manter-se } \\
\text { desperto) }\end{array}$ & $\begin{array}{l}\text { Espie } \\
2012^{35}\end{array}$ & Internet & $\begin{array}{c}\text { Classificação } \\
0-4\end{array}$ & 109 & $\begin{array}{c}-1,32 \\
{[-1,46 ;-1,18]}\end{array}$ & TCC \\
\hline $\begin{array}{l}\text { Performance diurna } \\
\text { (humor, relacionamentos, } \\
\text { energia) }\end{array}$ & $\begin{array}{l}\text { Espie } \\
2012^{35}\end{array}$ & Internet & $\begin{array}{c}\text { Classificação } \\
0-4\end{array}$ & 109 & {$[-1,64 ;-1,36]$} & TCC \\
\hline Latência do sono & $\begin{array}{l}\text { Espie } \\
2012^{35} \\
\text { Strom } \\
2004^{26} \\
\end{array}$ & Internet & $\begin{array}{l}\text { Diário de } \\
\text { sono }\end{array}$ & 190 & $\begin{array}{c}-25,34 \\
{[-59,52 ; 8,83]}\end{array}$ & TCC \\
\hline Latência do sono & $\begin{array}{l}\text { Van } \\
\text { Straten } \\
2009^{39}\end{array}$ & Autoaplicação & $\begin{array}{l}\text { Diário de } \\
\text { sono }\end{array}$ & 234 & $\begin{array}{c}-4,20 \\
{[-14,76 ; 6,36]}\end{array}$ & TCC \\
\hline Eficiência do sono & $\begin{array}{l}\text { Espie } \\
2012^{35} \\
\text { Strom } \\
2004^{26}\end{array}$ & Internet & $\begin{array}{l}\text { Diário de } \\
\text { sono }\end{array}$ & 190 & $\begin{array}{c}11,59 \\
{[-6,32 ; 29,50]}\end{array}$ & TCC \\
\hline Eficiência do sono & $\begin{array}{l}\text { Van } \\
\text { Straten } \\
2009^{39}\end{array}$ & Autoaplicação & $\begin{array}{l}\text { Diário de } \\
\text { sono }\end{array}$ & 234 & $\begin{array}{c}0,60 \\
{[-2,814,01]}\end{array}$ & TCC \\
\hline $\begin{array}{l}\text { Despertar após início do } \\
\text { sono }\end{array}$ & $\begin{array}{l}\text { Espie } \\
2012^{35} \\
\text { Strom } \\
2004^{26}\end{array}$ & Internet & $\begin{array}{l}\text { Diário de } \\
\text { sono }\end{array}$ & 190 & $\begin{array}{c}-24,91 \\
{[-76,02 ; 26,2]}\end{array}$ & TCC \\
\hline Redução de despertares & $\begin{array}{l}\text { Strom } \\
2004^{26}\end{array}$ & Internet & $\begin{array}{l}\text { Diário de } \\
\text { sono }\end{array}$ & 81 & 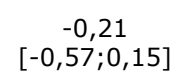 & TCC \\
\hline Tempo total de sono & $\begin{array}{l}\text { Espie } \\
2012^{35} \\
\text { Strom } \\
2004^{26} \\
\end{array}$ & Internet & $\begin{array}{l}\text { Diário de } \\
\text { sono }\end{array}$ & 190 & $\begin{array}{c}0,22 \\
{[-0.22 ; 0,66]}\end{array}$ & TCC \\
\hline Tempo total de sono & $\begin{array}{l}\text { Van } \\
\text { Straten } \\
2009^{39}\end{array}$ & Autoaplicação & $\begin{array}{l}\text { Diário de } \\
\text { sono }\end{array}$ & 234 & $\begin{array}{c}-0,19 \\
{[-0,49 ; 0,11]}\end{array}$ & Lista espera \\
\hline $\begin{array}{l}\text { Redução na frequência do } \\
\text { consumo de hipnóticos }\end{array}$ & $\begin{array}{l}\text { Strom } \\
2004^{26}\end{array}$ & Internet & $\begin{array}{l}\text { Número de } \\
\text { noites }\end{array}$ & 80 & $\begin{array}{c}-0,60 \\
{[-2,82 ; 1,62]}\end{array}$ & TCC \\
\hline $\begin{array}{l}\text { Redução na frequência do } \\
\text { consumo de hipnóticos }\end{array}$ & $\begin{array}{l}\text { Van } \\
\text { Straten } \\
2009^{39}\end{array}$ & Autoaplicação & $\begin{array}{l}\text { Número de } \\
\text { noites }\end{array}$ & 234 & $\begin{array}{c}0,10 \\
{[-1,07 ; 1,27]}\end{array}$ & TCC \\
\hline $\begin{array}{l}\text { Qualidade de vida (medida } \\
\text { pelo SF } 36 \text { ) }\end{array}$ & $\begin{array}{l}\text { Van } \\
\text { Straten } \\
2009^{39} \\
\end{array}$ & Autoaplicação & MCS & 247 & $\begin{array}{c}1,90 \\
{[0,40 ; 3,40]}\end{array}$ & TCC \\
\hline
\end{tabular}

Tabela 2. Resultados dos artigos incluídos. 


\begin{tabular}{|c|c|c|c|c|c|c|}
\hline \multicolumn{7}{|c|}{ Comparação TCC x Intervenções Farmacológicas } \\
\hline Desfecho ou subgrupo & Artigo & $\begin{array}{c}\text { Tipo de } \\
\text { aplicação da } \\
\text { TCC } \\
\end{array}$ & Medida & $\mathbf{N}$ & $\begin{array}{c}\text { Efeito } \\
\text { Estimado }\end{array}$ & $\begin{array}{c}\text { Desfecho } \\
\text { favorável para: }\end{array}$ \\
\hline $\begin{array}{l}\text { Performance - tempo de } \\
\text { reação (em segundos) }\end{array}$ & $\begin{array}{l}\text { Omvik } \\
2008^{37}\end{array}$ & \multirow{14}{*}{$\begin{array}{l}\text { Sessões } \\
\text { individuais }\end{array}$} & Vigil, 5.0 & 31 & $\begin{array}{c}0,00 \\
{[-0,06} \\
0,06]\end{array}$ & Sem diferença \\
\hline $\begin{array}{l}\text { Performance - número de } \\
\text { respostas corretas }\end{array}$ & $\begin{array}{l}\text { Omvik } \\
2008^{37}\end{array}$ & & Vigil, 5.0 & 31 & $\begin{array}{c}0,09 \\
{[-1,28} \\
1,46]\end{array}$ & TCC \\
\hline Performance - alerta & $\begin{array}{l}\text { Omvik } \\
2008^{37}\end{array}$ & & $\begin{array}{l}\text { Diário de } \\
\text { sono }\end{array}$ & 37 & $\begin{array}{c}-0,28 \\
{[-0,81} \\
0,25]\end{array}$ & I. Farmacológica \\
\hline Latência do sono & $\begin{array}{l}\text { Jacobs } \\
2004^{36}\end{array}$ & & $\begin{array}{l}\text { Diário de } \\
\text { sono }\end{array}$ & 25 & $\begin{array}{c}-24,60 \\
{[-54,12} \\
4,92]\end{array}$ & TCC \\
\hline Latência do sono & $\begin{array}{l}\text { Jacobs } \\
2004^{36}\end{array}$ & & $\begin{array}{c}\text { Sensor } \\
\text { movimento }\end{array}$ & 25 & $\begin{array}{c}-19,00 \\
{[-43,15} \\
5,15]\end{array}$ & TCC \\
\hline Eficiência do sono & $\begin{array}{l}\text { Sivertsen } \\
2006^{38}\end{array}$ & & PSG & 34 & $\begin{array}{c}7,40 \\
{[1,41 ;} \\
13,39]\end{array}$ & TCC \\
\hline Eficiência do sono & $\begin{array}{c}\text { Jacobs } \\
2004^{36} \\
\text { Sivertsen } \\
2006^{38} \\
\end{array}$ & & $\begin{array}{l}\text { Diário de } \\
\text { sono }\end{array}$ & 59 & $\begin{array}{l}12,02 \\
{[4,37} \\
19,67]\end{array}$ & TCC \\
\hline Eficiência do sono & $\begin{array}{l}\text { Jacobs } \\
2004^{36}\end{array}$ & & $\begin{array}{l}\text { Sensor } \\
\text { movimento }\end{array}$ & 25 & $\begin{array}{c}6,00 \\
{[-1,17} \\
13,17] \\
\end{array}$ & TCC \\
\hline Tempo total de sono & $\begin{array}{l}\text { Sivertsen } \\
2006^{38}\end{array}$ & & PSG & 34 & $\begin{array}{c}0,36 \\
{[-0,28} \\
1,00]\end{array}$ & TCC \\
\hline Tempo total de sono & $\begin{array}{c}\text { Jacobs } \\
2004^{36} \\
\text { Sivertsen } \\
2006^{38} \\
\end{array}$ & & $\begin{array}{l}\text { Diário de } \\
\text { sono }\end{array}$ & 61 & $\begin{array}{c}-0,16 \\
{[-0,72} \\
0,41]\end{array}$ & I. Farmacológica \\
\hline Tempo total de sono & $\begin{array}{l}\text { Jacobs } \\
2004^{36}\end{array}$ & & $\begin{array}{l}\text { Sensor } \\
\text { movimento }\end{array}$ & 25 & $\begin{array}{c}0,83 \\
{[-0,23} \\
1,89] \\
\end{array}$ & TCC \\
\hline Depressão & $\begin{array}{l}\text { Omvik } \\
2008^{37}\end{array}$ & & BDI & 38 & $\begin{array}{c}-1,62 \\
{[-5,15} \\
1,91]\end{array}$ & TCC \\
\hline $\begin{array}{l}\text { Qualidade de vida } \\
\text { (medida pelo SF } 36 \text { ) }\end{array}$ & $\begin{array}{l}\text { Omvik } \\
2008^{37}\end{array}$ & & PCS & 39 & $\begin{array}{c}1,97 \\
{[-3,90} \\
7,84]\end{array}$ & TCC \\
\hline $\begin{array}{l}\text { Qualidade de vida } \\
\text { (medida pelo SF } 36 \text { ) }\end{array}$ & $\begin{array}{l}\text { Omvik } \\
2008^{37}\end{array}$ & & MCS & 39 & $\begin{array}{c}0,31 \\
{[-6,12} \\
6,74]\end{array}$ & TCC \\
\hline \multicolumn{7}{|c|}{ Comparação TCC x Outras Intervenções Não Farmacológicas } \\
\hline Desfecho ou subgrupo & Artigo & $\begin{array}{c}\text { Tipo de } \\
\text { aplicação da } \\
\text { TCC }\end{array}$ & Medida & $\mathbf{N}$ & $\begin{array}{c}\text { Efeito } \\
\text { estimado }\end{array}$ & $\begin{array}{c}\text { Desfecho } \\
\text { favorável para: }\end{array}$ \\
\hline Melhor qualidade do sono & \multirow{8}{*}{ Espie $2012^{35}$} & \multirow{8}{*}{ Internet } & $\begin{array}{c}\text { Escala } 1 \text { a } \\
100\end{array}$ & 110 & $\begin{array}{c}4,30 \\
{[-1,87} \\
10,47]\end{array}$ & TCC \\
\hline Melhor qualidade do sono & & & SCI & 109 & $\begin{array}{c}1,82 \\
{[1,71 ;} \\
1,93]\end{array}$ & TCC \\
\hline $\begin{array}{l}\text { Performance diurna } \\
\text { (concentração, } \\
\text { produtividade, manter-se } \\
\text { desperto) }\end{array}$ & & & $\begin{array}{l}\text { Classificação } \\
0-4\end{array}$ & 110 & $\begin{array}{c}-0,63 \\
{[-0,78 ;-} \\
0,48]\end{array}$ & TCC \\
\hline $\begin{array}{l}\text { Performance diurna } \\
\text { (humor, relacionamentos, } \\
\text { energia) }\end{array}$ & & & $\begin{array}{c}\text { Classificação } \\
0-4\end{array}$ & 109 & $\begin{array}{c}-1,45 \\
{[-1,60 ;-} \\
1,30] \\
\end{array}$ & OINF \\
\hline Latência do sono & & & $\begin{array}{l}\text { Diário de } \\
\text { sono }\end{array}$ & 110 & $\begin{array}{c}-26,50 \\
{[-37,8 ;-} \\
15,2] \\
\end{array}$ & TCC \\
\hline Eficiência do sono & & & $\begin{array}{l}\text { Diário de } \\
\text { sono }\end{array}$ & 110 & $\begin{array}{l}11,90 \\
{[7,13} \\
16,67] \\
\end{array}$ & TCC \\
\hline $\begin{array}{l}\text { Despertar após início do } \\
\text { sono }\end{array}$ & & & $\begin{array}{l}\text { Diário de } \\
\text { sono }\end{array}$ & 110 & $\begin{array}{c}-26,30 \\
{[-39,9 ;-} \\
12,7] \\
\end{array}$ & TCC \\
\hline Tempo total de sono & & & $\begin{array}{l}\text { Diário de } \\
\text { sono }\end{array}$ & 110 & $\begin{array}{c}-0,11 \\
{[-0,61 ;} \\
0,39]\end{array}$ & OINF \\
\hline
\end{tabular}


acordado, o grupo de intervenção TCC apresentou melhora em relação ao grupo LE (MD -1,32; IC -1,46 a -1,18; $\mathrm{p}<0,00001$ ) e em relação aos grupos OINF, TCC (MD -0,63; IC $-0,78$ a $-0,48 ; \quad p<0,00001)$. Sobre humor, relacionamentos e energia, TCC apresentou melhora em relação ao grupo LE (MD -1,50; IC -1,64 a -1,36; p<0,00001) e ao grupo OINF (MD -1,45; IC -1,60 a -1,30; $p<0,00001)$. Apesar dos resultados favoráveis, a qualidade da evidência foi considerada muito baixa.

A redução nos sintomas subjetivos de insônia foi reportada por meio do Sleep Evaluation Form (SEF) (Van Straten 200939) e pela escala Dysfunctional Beliefs and Attitudes about Sleep (DBAS) (Strom $2004^{26}$ e Van Straten $2009^{39}$ ). TCC apresentou resultados favoráveis comparada a LE. Por meio do SEF, TCC apresentou MD 2,30 (IC 1,61 a $2,99 ; p<0,00001)$. Na escala DBAS, TCC obteve MD $-14,40$ (IC -23,13 a -5,67; $p=0,001$ ) (Strom 2004) 26 e MD 10,00 (IC 6,65 a 13,35; p<0,00001) (Van Straten 2009) ${ }^{39}$. A qualidade da evidência foi considerada muito baixa segundo a classificação do GRADE.

Os desfechos referentes ao número de despertares, o tempo total de sono, a latência do sono e despertares após início do sono foram mensurados por meio de diários de sono, polissonografia (Sivertsen 2006) (38 $^{38}$ em sensor de movimento (Jacobs 2004) ${ }^{36}$, mas não foram verificadas diferenças entre os grupos nessas variáveis.

Sivertsen $2006^{38}$ apresentou aumento na eficiência do sono no grupo de intervenção TCC comparado ao grupo de 
Intervenção Farmacológica (MD 7,40; IC 1,41 a 13,39; $p=0,02)$, com alta qualidade da evidência.

Juntos Sivertsen $2006^{38}$ e Jacobs $2004^{36}$ apresentaram o mesmo (MD 12,02; IC 4,37 a 19,67; $\mathrm{I}^{2}=0 \% ; \mathrm{p}=0,002$ ). Entretanto, a qualidade da evidência foi considerada muito baixa.

Ainda sobre o aumento na eficiência do sono, Espie $2012^{35}$ apresentou melhora para o grupo TCC e, comparação com o grupo OINF (MD 11,90; IC 7,13 a $16,67 ; \quad p<0,00001)$. A qualidade da evidência foi considerada muito baixa.

O desfecho de Qualidade de Vida foi mensurado por meio do SF-36, como previsto no protocolo (Omvik $2008^{37}$ e Van Straten $2009^{39}$ ). O grupo TCC apresentou melhora na Qualidade de Vida em comparação com o grupo LE (Van Straten 2009) ${ }^{39}$, pelo Mental Health Composite Score (MCS) (MD 1,$90 ;$ IC 0,40 a 3,$40 ; p=0,01$ ). A qualidade da evidência foi considerada moderada.

Nenhum dos artigos avaliou níveis de ansiedade pelo BAI, nem reportaram efeitos adversos da TCC.

\section{DISCUSSÃO}

Esta revisão demonstra que a TCC apresentou tendência favorável a ser superior à lista de espera (LE) em melhorar a qualidade do sono, melhorar a performance diurna em relação a concentração, produtividade, capacidade de manter-se acordado, humor, 
relacionamentos e energia (Espie 2012 ${ }^{35}$ ), em reduzir dos sintomas subjetivos da insônia (Strom 2004 ${ }^{26}$, Van Straten $2009^{39}$ ) e em melhorar a qualidade de vida (Van Straten $\left.2009^{39}\right)$.

Comparada aos grupos de Intervenção Farmacológica (IF), a TCC apresentou tendência favorável a ser superior apenas em aumentar a eficiência do sono, como relatado nos diários de sono e polissonografia (Jacobs $2004^{36}$ e Sivertsen $2006^{38}$ ). A análise do sensor de movimento não demonstrou diferença entre os grupos, o que sugere a necessidade de mais investigações sobre a validade desse instrumento de medida.

Comparada aos grupos de outras intervenções não farmacológicas, a TCC apresentou tendência favorável a ser superior em melhorar a qualidade do sono e a performance diurna em relação a concentração, produtividade, capacidade de manter-se acordado, humor, relacionamentos, energia, e em aumentar a eficiência do sono (Espie 2012 35 ).

Apesar de a TCC ser recomendada pela literatura para o tratamento da insônia, não há um protocolo validado que oriente quais são as técnicas que devem ser usadas obrigatoriamente. As técnicas da TCC foram combinadas de diversas maneiras nos estudos encontrados para esta revisão, o que dificultou a inclusão de estudos e aumentou o número de artigos excluídos que não satisfizeram as definições de protocolo. Foram identificados muitos artigos 
que utilizaram apenas uma ou duas das técnicas descritas no protocolo.

Além dos diferentes modelos de estudo, outros aspectos que dificultaram a análise e o agrupamento de dados e que podem ter afetado os resultados da metaanálise incluem a variedade de resultados escolhidos para serem avaliados nos estudos, o modo como as quatro técnicas selecionadas para o protocolo foram administradas (internet, sessões individuais, sessões de grupo, autoajuda) e o método para mensurar os desfechos escolhidos.

Os resultados favoráveis encontrados nesta revisão denotam a eficiência da TCC quando administrada de várias maneiras. Muitos grupos têm dedicado seu trabalho para estudar administração da TCC de modo online, em uma tentativa de aliviar a sobrecarga dos sistemas de saúde e democratizar o acesso da população ao atendimento, atualmente limitado, devido ao pequeno número de profissionais treinados. Os resultados destes estudos demonstram a melhoria na qualidade do sono, na eficiência do sono, no número de despertares e na diminuição da latência do sono ${ }^{17}$.

Foram incluídos na revisão estudos que utilizaram a TCC como uma combinação das técnicas de controle de estímulos, reestruturação cognitiva, restrição de sono e técnicas de relaxamento. Muitos estudos têm usado esse conjunto de técnicas, exceto as técnicas de relaxamento. Esses estudos não foram incluídos, e outra avaliação é recomendada para analisar esses ensaios. 
A higiene do sono é considerada pelos Guidelines como uma técnica associada à $\operatorname{TCC}^{13}$, e, nos estudos incluídos, ela foi combinada às intervenções em todos os grupos. Como uma técnica independente, a higiene do sono foi utilizada como grupo de controle, e, assim, foi considerado neste estudo como parte da classificação OINF de grupos de controle.

Em geral, os resultados dos desfechos dos estudos incluídos tendenciam o uso da TCC para o tratamento da insônia, embora algumas comparações não tenham apresentado diferenças significativas entre os grupos controle e os grupos de intervenção.

Questões importantes relativas a possíveis diferenças na adesão ao tratamento por homens e mulheres, insones agudos ou crônicos, jovens e adultos insones contra idosos devem ser exploradas no futuro.

Outro fator importante é que a qualidade das evidências foi considerada muito baixa, o que pressupõe que o tratamento da TCC deva ser alvo de mais investigações para que possa ser replicado na população em geral, uma vez que estas conclusões são baseadas em apenas um ou dois artigos com poucos participantes.

Algumas análises apresentaram grande heterogeneidade, 0 que pode ser justificado pela diversidade dos meios de aplicação das intervenções nos estudos e pela diferença de idade dos participantes.

Apesar dos resultados clínicos favoráveis apresentados, a TCC não demonstrou impacto em muitos 
desfechos. Por conta disso, novas pesquisas devem ser realizadas, principalmente ensaios clínicos randomizados com amostras representativas, para que a TCC possa ser recomendada para a melhora desses desfechos no tratamento da insônia primária.

Há poucas revisões sistemáticas de ensaios clínicos randomizados que avaliem a TCC e os grupos controle conforme determina este protocolo.

Schwartz $2012^{40}$ revisou estudos que avaliaram a TCC e incluiu 21 ensaios clínicos randomizados (agrupou diversas técnicas e grupos controle). Os resultados relacionados ao tempo de leito, cochilos e número de despertares demonstraram resultados significantes à TCC. Entretanto, estudos com pacientes com comorbidades ou insônia secundária não foram excluídos.

Mitchell $2012^{41}$ avaliou cinco artigos que avaliaram a TCC comparada a medicações prescritas e não prescritas à pacientes com insônia primária. Os desfechos avaliados foram o tempo total de sono, latência do sono, eficiência do sono e tempo total de vigília após o início do sono. Os resultados foram avaliados a curto e longo prazo, e ao contrário do que se apresente nesta revisão o tratamento medicamentoso aumentou a eficiência do sono, reduziu o tempo de latência, e aumentou o tempo total de sono comparado à TCC. O objetivo desta revisão não foi o de avaliar os desfechos em longo prazo. Mitchell $2012^{41}$ afirma que a TCC é mais eficaz que os medicamentos benzodiazepínicos e não benzodiazepínicos em longo prazo 
(qualidade da evidência de baixa a moderada), e que os benzodiazepínicos são mais eficazes que a TCC em curto prazo (qualidade da evidência muito baixa).

Várias questões metodológicas precisam ser abordadas ao considerar os achados desta revisão. A TCC tem validade clínica, como demonstram muitas séries de caso e ensaios clínicos, e embora a TCC tenha demonstrado superioridade em relação aos grupos controle nos estudos incluídos, muitos resultados não diferiram entre os grupos, sugerindo que estudos bem desenhados adicionais com grandes amostras ainda são necessários para avaliar o efeito desta intervenção.

Os desfechos mais favoráveis à TCC foram encontrados nas comparações aos grupos de lista de espera. Esse tipo de grupo controle parece ser adequado para comparação, pois os pacientes podem ser monitorados sistematicamente enquanto aguardam pelo tratamento.

São comuns os resultados das comparações da TCC com outras intervenções serem menos expressivos do que em comparação com a lista de espera. Provavelmente essa diferença se deve ao vínculo estabelecido entre paciente e terapeuta. Esses pacientes recebem de certa forma algum cuidado enquanto aguardam o atendimento em TCC, o que deve promover mudanças nos hábitos de sono e até na percepção do sono, além da diminuição da ansiedade.

Os estudos primários incluídos administraram intervenções de TCC de maneiras diferentes. Essas variações são observadas na gestão dos pacientes, na 
aplicação em grupo vs. sessões individuais e no formato de atendimento (pessoalmente, via internet ou por meio de livros ou folhetos). A quantidade, a frequência e o tempo das visitas também diferiram muito entre os estudos, e essa fonte de viés pode influenciar a interpretação dos resultados.

A falta de um protocolo para ensaios clínicos que visam medir os benefícios da TCC aplicada à insônia dificulta a inclusão de muitos estudos, devido a dados incompletos ou insuficientes. Na maioria dos estudos, as técnicas de TCC foram administradas face a face, e os grupos de controle foram muito diferentes dos grupos de TCC. Isso dificulta controlar o viés no cegamento de participantes e terapeutas.

Como esta revisão teve como objetivo avaliar a eficiência da TCC em curto prazo, os resultados obtidos durante o follow-up (geralmente em 3-6 meses) não foram avaliados. Nós não verificamos a durabilidade de melhora após o tratamento; e esse pode ser considerado um viés desfavorável para protocolos que avaliem TCC, uma vez que a terapia é uma intervenção eficaz em longo prazo ${ }^{42}$.

Para avaliar a qualidade de evidência da TCC, as técnicas de TCC empregadas devem ser avaliadas conforme sua eficácia. A padronização é também necessária na medição de resultados, por exemplo, em relação ao uso de escalas, diários de sono e polissonografia. A dificuldade de criar um protocolo eficiente pode ser resultado da diversidade das demandas de tratamento em toda a 
população, sendo que alguns pacientes necessitam de intervenções mais cognitivas, enquanto outros exigem intervenções comportamentais.

A construção e validação de um protocolo para orientar a escolha das intervenções para tratamento de TCC na insônia deve ser uma prioridade nas pesquisas futuramente. No que diz respeito à concepção desses estudos futuros, recomendamos estudos randomizados controlados de TCC que consistam em uma combinação de controle de estímulos, reestruturação cognitiva, restrição do sono e técnicas de relaxamento, incluindo os métodos de higiene do sono.

Sugerimos também que a mensuração dos resultados deva ser padronizada por polissonografia, diários de sono e Pittsburgh Sleep Quality Index (PSQI) ${ }^{43}$ para os resultados relacionados ao sono, BDI para avaliar sintomas depressivos e BAI para sintomas de ansiedade. Recomendamos que o SF-36 seja usado para medir as mudanças na qualidade de vida.

Ensaios clínicos com amostras maiores são necessários para aumentar o poder estatístico dos resultados. Há também uma necessidade de novas revisões para avaliar os resultados observados nos follow-ups, permitindo, assim, a investigação dos efeitos das intervenções de TCC para a insônia, em longo prazo. 


\section{CONCLUSÕES}

Dados os resultados desta revisão, a TCC pode ser indicada para melhorar a qualidade de vida e a atividade diurna, e também para aumentar a eficiência do sono em pacientes insones. Embora os resultados demonstrem tendências favoráveis ao uso da TCC, são necessárias mais investigações. A qualidade das evidências foi considerada como muito baixa, e a maioria dos resultados foi avaliada em relação a apenas um artigo, não sendo possível a obtenção de meta-análises.

\section{AGRADECIMENTOS}

Agradecemos a CAPES (SESU/REUNI) pelo apoio.

\section{REFERÊNCIAS}

1.AASM. International Classification of Sleep Disorders: Diagnostic and Coding Manual, 3rd ed. Darien: American Academy of Sleep Medicine, 2014.

2.Partinen M, Hublin C. Epidemiology of Sleep Disorders. In: Kryger MH, Roth T, Dement WC, eds. Principles and Practice of Sleep Medicine. Philadelphia: WB Saunders Company, 2000, p558-79.
3.Buysse
DJ.
Insomnia.
JAMA 2013;309:706-16. https://doi.org/10.1001/jama.2013.193

4.Edinger JD, Wohlgemuth WK, Radtke RA, Marsh GR, Quillian RE. Cognitive Behavioral Therapy for Treatment of Chronic Primary Insomnia: A Randomized Controlled Trial. JAMA 2001;285:1856-64. https://doi.org/10.1001/jama.285.14.1856

5.Morin CM, Colecchi C, Stone J, Sood R, Brink D. Behavioral and Pharmacological Therapies for Late-Life Insomnia: A Randomized Controlled Trial. JAMA 1999;281:991-9. https://doi.org/10.1001/jama.281.11.991

6. Morgan K, Dixon S, Mathers N, Thompson J, Tomeny M. Psychological treatment for insomnia in the management of long- 
term hypnotic drug use: a pragmatic randomized controlled trial. $\mathrm{Br}$ ] Gen Pract 2003;53:923-8.

7.Stepanski E. Behavioral Therapy for Insomnia. In: Kryger MH, Roth $\mathrm{T}$, Dement WC (eds). Principles and Practice of Sleep Medicine. Philadelphia: WB Saunders Company, 2000, 647-56.

8.Jansson K, Linton SJ. Cognitive-behavioral group therapy as an early intervention for insomnia: a randomized controlled trial. J Occup Rehabil 2005;15:177-90.

9.Morin CM, Culbert JP, Schwartz SM. Nonpharmachological interventions for insomnia: a meta-analysis of treatment efficacy. Am J Psychiatry 1994;151:1172-80.

https://doi.org/10.1176/ajp.151.8.1172

10. Morin CM, Blais F, Savard J. Are changes in beliefs and attitudes about sleep related to sleep improvements in the treatment of insomnia? Behav Res Ther 2002;40:741-52. https://doi.org/10.1016/s0005-7967(01)00055-9

11.Baillargeon L. Behavior and cognitive treatments for insomnia. An alternative to pharmacotherapy. Can Fam Physician 1997;43:290-6.

12.Baillargeon L, Landreville P, Verreault R, Beauchemin JP, Gregoire JP, Morin CM. Discontinuation of benzodiazepines among older insomnia adults treated with cognitive-behavioural therapy combined gradual tapering: a randomised trial. CMAJ 2003;169:1015-20.

13.Schutte-Rodin S, Broch L, Buysse D, Dorsey C, Sateia M. Clinical Guideline for the Evaluation and Management of Chronic Insomnia in Adults. J Clin Sleep Med 2008;4:487-504.

14. Yamadera W, Sato M, Harada D, Iwashita M, Aoki R, Obuchi K, et al. Comparisons of short-term efficacy between individual and group cognitive behavioral therapy for primary insomnia. Sleep Biol Rhythms 2013;11:176-84. https://doi.org/10.1111/sbr.12019

15.Koffel EA, Koffel JB, Gehrman PR. A meta-analysis of group cognitive behavioral therapy for insomnia. Sleep Med Rev 2015;19:616. https://doi.org/10.1016/j.smrv.2014.05.001

16. Ho FY, Chung KF, Yeung WF, Ng TH, Kwan KS, Yung KP, et al. Self-help cognitive-behavioral therapy for insomnia: A meta-analysis of randomized controlled trials. Sleep Med Rev 2015; 19:1728. https://doi.org/10.1016/j.smrv.2014.06.010

17.Cheng SK, Dizon J. Computerised cognitive behavioural therapy for insomnia: a systematic review and meta-analysis. Psychother Psychosom 2012;81:206-16. https://doi.org/10.1159/000335379

18. Higgins JPT, Green S (eds). Cochrane Handbook for Systematic Reviews of Interventions Version 5.1.0. The Cochrane Collaboration, 2011. Disponível em: www.cochrane-handbook.org

19. American Psychiatric Association. Diagnostic and Statistical Manual of Mental Disorders (DSM-IV). 4th ed. Washington: American Psychiatric Association, 1994.

20.World Health Organization. International Statistical Classification of Diseases and Related Health Problems. Tenth Revision Geneva: World Health Organization, 1992. 
21.Zarcone VP. Sleep hygiene. In: Kryger MH, Roth T, Dement WC (eds). Principles and Practice of Sleep Medicine. Philadelphia: WB Saunders Company, 2000:657-61.

22. Morin C. Psychological and behavioral treatments for primary insomnia. In: Kryger $\mathrm{MH}$, Roth $\mathrm{T}$, Dement WC (eds). Principles and Practice of Sleep Medicine. 4 ed. Philadelphia: Elsevier /WB Saunders Company, 2005:726-37.

23.Smith MT, Neubauer DN. Cognitive behavior therapy for chronic insomnia. Clin Cornerstone 2003;5:28-40. https://doi.org/10.1159/000335379

24.Bastien CH, Morin CM, Ouellet MC, Blais FC, Bouchard S. Cognitive-behavioral therapy for insomnia: comparison of individual therapy, group therapy, and telephone consultations. J Consult Clin Psychol 2004;72:653-9. https://doi.org/10.1037/0022-006X.72.4.653 25. Waters WF, Hurry MJ, Binks PG, Carney CE, Lajos LE, Fuller KH, et al. Behavioral and hypnotic treatments for insomnia subtypes. Behav Sleep Med 2003;1:81-101.

https://doi.org/10.1207/S15402010BSM0102 2

26.Strom L, Pettersson R, Andersson G. Internet-Based Treatment for Insomnia: A Controlled Evaluation. J Consult Clin Psychol 2004;72:113-20.

https://doi.org/10.1037/0022-006X.72.1.113

27.Beck AT, Epstein N, Brown G. An inventory for measuring clinical anxiety: psychometric properties. J Consult Clin Psychol 1988;56:893-7. $\quad$ https://doi.org/10.1037//0022$006 \times \cdot 56.6 .893$

28.Beck AT, Ward CH, Mendelson M, Mock J, Erbaugh J. An inventory for measuring depression. Arch Gen Psychiatry 1961;4:561-71. https://doi.org/10.1001/archpsyc.1961.01710120031004

29. Ware JE, Snow KK, Kosinski M, Gandek B. SF-36 Manual and Interpretation Guide. Boston, MA: New England Medical Center, 1993. 30.Arnedt JT, Cuddihy L, Swanson LM, Pickett S, Aikens J, Chervin RD. Randomized Controlled Trial of Telephone-Delivered Cognitive Behavioral Therapy for Chronic Insomnia. Sleep 2013;36:353-62. https://doi.org/10.5665/sleep.2448

31.Bjorvatn B, Fiske E, Pallesen S. A self-help book is better than sleep hygiene advice for insomnia: A randomized controlled comparative study. Scand J Psychol 2011;52:580-5. https://doi.org/10.1111/j.1467-9450.2011.00902.x

32.Espie CA, Inglis SJ, Tessier S, Harvey L. The clinical effectiveness of cognitive behaviour therapy for chronic insomnia: implementation and evaluation of a sleep clinic in general medical practice. Behav Res Ther 2001;39:45-60. https://doi.org/10.1016/s00057967(99)00157-6

33.Fornal-Pawłowska M, Szelenberger W. Cognitive behavioral therapy for chronic insomnia. Psychiatria Polska 2013;47:269-277. 34. Ueda M, Adachi Y, Hayama J, Yamagami T. Preparation and effect of a behavioral science-based education program for sleep 
improvement among medical students. Jap J Public Health 2008;55:3-10.

35.Espie CA, Kyle SD, Williams C, Ong JC, Douglas NJ, Hames P, et al. A Randomized, Placebo-Controlled Trial of Online Cognitive Behavioral Therapy for Chronic Insomnia Disorder Delivered via an Automated Media-RichWeb Application. Sleep 2012;35:769-81. https://doi.org/10.5665/sleep.1872

36.Jacobs GD, Pace-Schott EF, Stickgold R, Otto MW. Cognitive Behavior therapy and pharmacotherapy for insomnia. Arch Intern Med 2004;164:1888-96.

https://doi.org/10.1001/archinte.164.17.1888

37.Omvik S, Sivertsena B, Pallesen S, Bjorvatn B, Havik OE, Nordhus IH. Daytime functioning in older patients suffering from chronic insomnia: Treatment outcome in a randomized controlled trial comparing CBT with Zopiclone. Behav Res Ther 2008;46:623-41. https://doi.org/10.1016/j.brat.2008.02.013

38. Sivertsen B, Omvik S, Pallensen S, Bjorvatn B, Havik OE, Kvale G, et al. Cognitive Behavioral Therapy vs Zopiclone for Treatment of Chronic Primary Insomnia in Older Adults: A Randomized Controlled Trial. JAMA 2006;295:2851-8.

https://doi.org/10.1001/jama.295.24.2851

39.Van Straten A, Cuijpers P, Smit F, Spermon M, Verbeek I. Selfhelp treatment for insomnia through television and book: $A$ randomized trial. Patient Educ Couns 2009;74:29-34. https://doi.org/10.1016/j.pec.2008.07.050

40.Schwartz DR, Carney CE. Mediators of cognitive-behavioral therapy for insomnia: a review of randomized controlled trials and secondary analysis studies. Clin Psychol Rev 2012;32:664-75. https://doi.org/10.1016/j.cpr.2012.06.006

41. Mitchell MD, Gehrman P, Perlis P, Umscheid CA. Comparative effectiveness of cognitive behavioral therapy for insomnia: a systematic review. BMC Family Practice 2012;13:40. https://doi.org/10.1186/1471-2296-13-40

42. Montgomery $P$, Dennis J. Cognitive behavioural interventions for sleep problems in adults aged $60+$ (Cochrane Review). Cochrane Database Syst Rev 2003;(1):CD003161. https://doi.org/10.1002/14651858.CD003161

43. Buysse DJ, Reynolds CF 3rd, Monk TH, Berman SR, Kupfer DJ. The Pittsburgh Sleep Quality Index (PSQI): A new instrument for psychiatric research and pratice. Psychiatr Res 1989;28:193-213. https://doi.org/10.1016/0165-1781(89)90047-4 